Kragujevac Journal of Mathematics

Volume 45(5) (2021), Pages 815-827.

\title{
ON $\check{\phi}$-SEMISYMMETRIC $L P$-KENMOTSU MANIFOLDS WITH A QSNM-CONNECTION ADMITTING RICCI SOLITONS
}

\author{
RAJENDRA PRASAD ${ }^{1}$, ABDUL HASEEB $^{2}$, AND UMESH KUMAR GAUTAM $^{1}$ \\ ABSTRACT. In the present work, we characterize Lorentzian para-Kenmotsu (briefly, \\ $L P$-Kenmotsu) manifolds with a quarter-symmetric non-metric connection (briefly, \\ QSNM-connection) $\hat{\nabla}$ satisfying certain $\ddot{\phi}$-semisymmetric conditions admitting Ricci \\ solitions. At the end of the paper, a 3-dimensional example of $L P$-Kenmotsu \\ manifolds with a connection $\widehat{\nabla}$ is given to verify some results of the present paper.
}

\section{Introduction}

In a $(2 n+1)$-dimensional connected and $C^{\infty}$-smooth semi-Riemannian manifold $(M, \check{g})$, the Levi-Civita connection $\check{\nabla}$, the Riemannian-Christoffel curvature tensor $\check{R}$, the projective curvature tensor $\check{P}$, the concircular curvature tensor $\check{V}$, the conformal curvature tensor $\check{C}$ and the $D$-conformal curvature tensor $\check{B}$ are defined by $[5,6]$

$$
\check{R}(\check{E}, \check{F}) \check{W}=\check{\nabla}_{\check{E}} \check{\nabla}_{\check{F}} \check{W}-\check{\nabla}_{\check{F}} \check{\nabla}_{\check{E}} \check{W}-\check{\nabla}_{[\check{E}, \check{F}]} \check{W},
$$

$$
\check{P}(\check{E}, \check{F}) \check{W}=\check{R}(\check{E}, \check{F}) \check{W}-\frac{1}{2 n}[\check{S}(\check{F}, \check{W}) \check{E}-\check{S}(\check{E}, \check{W}) \check{F}]
$$

$$
\check{V}(\check{E}, \check{F}) \check{W}=\check{R}(\check{E}, \check{F}) \check{W}-\frac{\check{r}}{2 n(2 n+1)}[\check{g}(\check{F}, \check{W}) \check{E}-\check{g}(\check{E}, \check{W}) \check{F}]
$$

Key words and phrases. LP-Kenmotsu manifold, QSNM-connection, $\check{\phi}$-semisymmetric manifolds, Ricci solitons.

2010 Mathematics Subject Classification. Primary: 53D15. Secondary: 53C05, 53C25.

DOI 10.46793/KgJMat2105.815P

Received: December 08, 2018.

Accepted: May 22, 2019. 


$$
\begin{aligned}
\check{C}(\check{E}, \check{F}) \check{W}= & \check{R}(\check{E}, \check{F}) \check{W}-\frac{1}{(2 n-1)}[\check{S}(\check{F}, \check{W}) \check{E}-\check{S}(\check{E}, \check{W}) \check{F} \\
& +\check{g}(\check{F}, \check{W}) \check{Q} \check{E}-\check{g}(\check{E}, \check{W}) \check{Q} \check{F}]+\frac{\check{r}}{2 n(2 n-1)}[\check{g}(\check{F}, \check{W}) \check{E}-\check{g}(\check{E}, \check{W}) \check{F}],
\end{aligned}
$$

$$
\begin{aligned}
\check{B}(\check{E}, \check{F}) \check{W}= & \check{R}(\check{E}, \check{F}) \check{W}+\frac{1}{2(n-1)}[\check{S}(\check{E}, \check{W}) \check{F}-\check{S}(\check{F}, \check{W}) \check{E}+\check{g}(\check{E}, \check{W}) \check{Q} \check{F} \\
& -\check{g}(\check{F}, \check{W}) \check{Q} \check{E}-\check{S}(\check{E}, \check{W}) \check{\eta}(\check{F}) \xi+\check{S}(\check{F}, \check{W}) \check{\eta}(\check{E}) \xi-\check{\eta}(\check{E}) \check{\eta}(\check{W}) \check{Q} \check{F} \\
& +\check{\eta}(\check{F}) \check{\eta}(\check{W}) \check{Q} \check{E}]-\frac{k-2}{2(n-1)}[\check{g}(\check{E}, \check{W}) \check{F}-\check{g}(\check{F}, \check{W}) \check{E}]+\frac{k}{2(n-1)} \\
& \times[\check{g}(\check{E}, \check{W}) \check{\eta}(\check{F}) \xi-\check{g}(\check{F}, \check{W}) \check{\eta}(\check{E}) \xi+\check{\eta}(\check{E}) \check{\eta}(\check{W}) \check{F}-\check{\eta}(\check{F}) \check{\eta}(\check{W}) \check{E}],
\end{aligned}
$$

respectively, where $\check{r}$ is the scalar curvature, $\check{S}$ and $\check{Q}$ are the Ricci tensor and the Ricci operator, respectively such that $\check{S}(\check{E}, \breve{F})=\check{g}(\check{Q} \breve{E}, \check{F})$ and $k=\frac{\check{r}+4 n}{2 n-1}$.

The connection $\widehat{\nabla}$ which is linear and defined on $(M, \check{g})$ is said to be a quartersymmetric [11] if its torsion tensor $\check{T}$

$$
\check{T}(\check{E}, \check{F})=\widehat{\nabla}_{\check{E}} \check{F}-\widehat{\nabla}_{\check{F}} \check{E}-[\check{E}, \check{F}]=\check{\eta}(\check{F}) \check{\phi} \check{E}-\check{\eta}(\check{E}) \check{\phi} \check{F}
$$

where $\check{\phi}$ is a $(1,1)$-tensor field and $\check{\eta}$ is a 1 -form. If moreover, $\widehat{\nabla}$ satisfies the condition

$$
\left(\widehat{\nabla}_{\check{E}} \check{g}\right)(\check{F}, \check{W})=-\check{\eta}(\check{F}) \check{g}(\check{\phi} \check{E}, \check{W})-\check{\eta}(\check{W}) \check{g}(\check{F}, \check{\phi} \check{E}),
$$

where $\check{E}, \check{F}, \check{W} \in \chi(M)$ and $\chi(M)$ is the set of all differentiable vector fields on $M$, then connection $\widehat{\nabla}$ is called a QSNM-connection. The authors in $[2,3,7,12]$ have studied QSNM-connection in various manifolds.

In an $L P$-Kenmotsu manifold, a relation between the connections $\widehat{\nabla}$ and $\check{\nabla}$ is given by

$$
\widehat{\nabla}_{\check{E}} \check{F}=\check{\nabla}_{\check{E}} \check{F}+\check{\eta}(\check{F}) \check{\phi} \check{E} .
$$

On a Riemannian manifold $(M, \check{g})$, a Ricci soliton $(\check{g}, U, \check{\lambda})$ is a generalization of an Einstein metric such that (see $[9,10]) \check{£}_{U} \check{g}+2 \check{S}+2 \check{\lambda} \check{g}=0$, where $\check{S}, \check{£}_{U}$ and $\check{\lambda}$ are the Ricci tensor, the Lie derivative operator along the vector field $U$ on $M$ and a real constant, respectively. A Ricci soliton is said to be shrinking, steady or expanding according as $\check{\lambda}<0, \check{\lambda}=0$ or $\check{\lambda}>0$, respectively.

The present work is arranged in the following manner. After Introduction, a brief introducton of LP-Kenmotsu manifolds is given in Section 2. In Section 3, we find the relation between the curvature tensors of an $L P$-Kenmotsu manifold with the connections $\check{\nabla}$ and $\widehat{\nabla}$. In Section 4, we study $L P$-Kenmotsu manifolds with a connection $\widehat{\nabla}$ admitting Ricci solitons. $\check{\phi}$-projectively semisymmetric, $\check{\phi}$-concircularly semisymmetric, $\check{\phi}$-conformally semisymmetric and $\check{\phi}$ - $D$-conformally semisymmetric $L P$-Kenmotsu manifolds with a connection $\widehat{\nabla}$ admitting Ricci solitons have been 
studied in Section 5. At the end of the paper, a 3-dimensional example of LPKenmotsu manifolds with a connection $\widehat{\nabla}$ is given to verify some results of the present paper.

\section{Preliminaries}

A $(2 n+1)$-dimensionsional differentiable manifold $M$ with structure $(\check{\phi}, \xi, \check{\eta}, \check{g})$ is said to be a Lorentzian almost paracontact metric manifold, if it admits $\phi$ : a tensor field of type $(1,1), \xi$ : a contravariant vector field, $\check{\eta}$ : a 1 -form and $\check{g}$ : a Lorentzian metric satisfying $[8]$

$$
\begin{aligned}
\check{\eta}(\xi) & =-1, \\
\check{\phi} 2 \check{E} & =\check{E}+\check{\eta}(\check{E}) \xi, \\
\check{\phi} \xi & =0, \quad \check{\eta}(\check{\phi} Y)=0, \\
\check{g}(\check{\phi} \check{E}, \check{\phi} \check{F}) & =\check{g}(\check{E}, \check{F})+\check{\eta}(\check{E}) \check{\eta}(\check{F}), \\
\check{g}(\check{E}, \xi) & =\check{\eta}(\check{E}), \\
\check{\Phi}(\check{F}, \check{E}) & =\check{\Phi}(\check{E}, \check{F})=\check{g}(\check{E}, \check{\phi} \check{F}),
\end{aligned}
$$

for any $\check{E}, \check{F}$ on $M$.

For $\xi$ : a killing vector field, the (para) contact structure is said to be a $K$-(para) contact. In this case, we have

$$
\check{\nabla}_{\check{E}} \xi=\check{\phi} \check{E} .
$$

A Lorentzian almost paracontact manifold $M$ is called an $L P$-Sasakian manifold if

$$
\left(\check{\nabla}_{\check{E}} \check{\phi}\right) \check{F}=\check{g}(\check{E}, \check{F}) \xi+\check{\eta}(\check{F}) \check{E}+2 \check{\eta}(\check{E}) \check{\eta}(\check{F}) \xi
$$

for any $\check{E}, \check{F}$ on $M$.

Now, we define a new manifold called a Lorentzian para-Kenmostu (briefly, LPKenmotsu) manifold:

Definition 2.1. A Lorentzian almost paracontact manifold is called Lorentzian paraKenmostu (briefy, LP-Kenmostu) manifold if [1]

$$
\left(\check{\nabla}_{\check{E}} \check{\phi}\right) \check{F}=-\check{g}(\check{\phi} \check{E}, \check{F}) \xi-\check{\eta}(\check{F}) \check{\phi} \check{E}
$$

for any $\check{E}, \check{F}$ on $M$.

In the Lorentzian para-Kenmostu manifold, we have

$$
\begin{aligned}
\check{\nabla}_{\check{E}} \xi & =-\check{\phi}^{2} \check{E}, \\
\left(\check{\nabla}_{\check{E}} \check{\eta}\right) \check{F} & =-\check{g}(\check{\phi} \check{E}, \check{\phi} \check{F}) .
\end{aligned}
$$

Moreover, on an LP-Kenmotsu, the following relations hold [1]:

$$
\check{g}(\check{R}(\check{E}, \check{F}) \check{W}, \xi)=\check{\eta}(\check{R}(\check{E}, \check{F}) \check{W})=\check{g}(\check{F}, \check{W}) \check{\eta}(\check{E})-\check{g}(\check{E}, \check{W}) \check{\eta}(\check{F})
$$




$$
\begin{aligned}
\check{R}(\xi, \check{E}) \check{F} & =-\check{R}(\check{E}, \xi) \check{F}=\check{g}(\check{E}, \check{F}) \xi-\check{\eta}(\check{F}) \check{E} \\
\check{R}(\check{E}, \check{F}) \xi & =\check{\eta}(\check{F}) \check{E}-\check{\eta}(\check{E}) \check{F} \\
\check{R}(\xi, \check{E}) \xi & =\check{E}+\check{\eta}(\check{E}) \xi \\
\check{S}(\check{E}, \xi) & =(\operatorname{dim} M-1) \check{\eta}(\check{E}), \quad \check{S}(\xi, \xi)=-(\operatorname{dim} M-1), \\
\check{Q} \xi & =(\operatorname{dim} M-1) \xi
\end{aligned}
$$

for any $\check{E}, \check{F}, \check{W}$ on $M$.

Definition 2.2. An $L P$-Kenmotsu manifold is called an $\eta$-Einstein manifold if its Ricci tensor satisfies [4] $\check{S}(\check{E}, \check{F})=a_{1} \check{g}(\check{E}, \check{F})+a_{2} \check{\eta}(\check{E}) \check{\eta}(\check{F})$, where $a_{1}$ and $a_{2}$ are smooth functions on $M$.

3. Curvature Tensor of LP-Kenmotsu Manifolds with a Connection $\widehat{\nabla}$

The curvature tensor $\widehat{R}$ of an $L P$-Kenmotsu manifold with a connection $\widehat{\nabla}$ is defined by

$$
\widehat{R}(\check{E}, \check{F}) \check{W}=\widehat{\nabla}_{\check{E}} \widehat{\nabla}_{\check{F}} \check{W}-\widehat{\nabla}_{\check{F}} \widehat{\nabla}_{\check{E}} \check{W}-\widehat{\nabla}_{[\check{E}, \check{F}]} \check{W} .
$$

From (1.8), (2.1), (2.4), (2.6), (2.7) and (3.1), we obtain

$$
\widehat{R}(\check{E}, \check{F}) \check{W}=\check{R}(\check{E}, \check{F}) \check{W}-\check{g}(\check{E}, \check{W}) \check{\phi} \check{F}+\check{g}(\check{F}, \check{W}) \check{\phi} \check{E},
$$

where $\check{R}(\check{E}, \check{F}) \check{W}$ is given by (1.1). Contracting $\check{E}$ in (3.2), we get

$$
\widehat{S}(\check{F}, \check{W})=\check{S}(\check{F}, \check{W})+\check{g}(\check{F}, \check{W}) \check{\psi}-\check{g}(\check{\phi} \check{F}, \check{W}) \text {. }
$$

From (3.3), it follows that

$$
\widehat{Q} \check{F}=\check{Q} \check{F}+\check{\psi} \check{F}-\check{\phi} \check{F}
$$

Contracting again $\check{F}$ and $\check{W}$ in (3.3), we obtain

$$
\widehat{r}=\check{r}+2 n \check{\psi},
$$

where $\widehat{Q}$ is the Ricci operator, $\widehat{S}$ is the Ricci tensor and $\widehat{r}$ is the scalar curvature with respect to $\widehat{\nabla}$.

Lemma 3.1. In a $(2 n+1)$-dimensional LP-Kenmotsu manifold with a connection $\widehat{\nabla}$, we have

$$
\begin{aligned}
\widehat{R}(\check{E}, \check{F}) \xi & =\check{\eta}(\check{F}) \check{E}-\check{\eta}(\check{E}) \check{F}+\check{\eta}(\check{F}) \check{\phi} \check{E}-\check{\eta}(\check{E}) \check{\phi} \check{F}, \\
\widehat{R}(\xi, \check{E}) \check{F} & =-\widehat{R}(\check{E}, \xi) \check{F}=\check{g}(\check{E}, \check{F}) \xi-\check{\eta}(\check{F}) \check{E}-\check{\eta}(\check{F}) \check{\phi} \check{E}, \\
\widehat{R}(\xi, \check{E}) \xi & =\check{\eta}(\check{E}) \xi+\check{E}+\check{\phi} \check{E}, \\
\widehat{S}(\check{E}, \xi) & =(2 n+\check{\psi}) \check{\eta}(\check{E}), \quad \widehat{S}(\xi, \xi)=-(2 n+\check{\psi}), \\
\widehat{\nabla}_{\check{E}} \xi & =-\check{E}-\check{\eta}(\check{E}) \xi-\check{\phi} \check{E}, \\
\widehat{Q} \xi & =(2 n+\check{\psi}) \xi,
\end{aligned}
$$


for any $\check{E}, \check{F}$ on $M$.

4. Ricci SOLIton on LP-Kenmotsu Manifolds With a CONNECtion $\widehat{\nabla}$

Suppose that an $L P$-Kenmotsu manifold with a connection $\widehat{\nabla}$ admits a Ricci soliton $(\check{g}, \xi, \check{\lambda})$. Then in view of (1.9), we have

$$
\left(\widehat{\check{£}}_{\xi} \breve{g}\right)(\check{F}, \breve{W})+2 \widehat{S}(\check{F}, \breve{W})+2 \check{\lambda} \check{g}(\check{F}, \breve{W})=0 .
$$

By using (3.7) and (1.6), we find

$$
\left(\check{\check{E}}_{\xi} \check{g}\right)(\check{F}, \check{W})=-2[\check{g}(\check{F}, \check{W})+\check{\eta}(\check{F}) \check{\eta}(\check{W})] .
$$

Combining (4.1) and (4.2), we obtain

$$
\widehat{S}(\check{F}, \check{W})=(1-\check{\lambda}) \check{g}(\check{F}, \check{W})+\check{\eta}(\check{F}) \check{\eta}(\check{W}) .
$$

Taking $\check{W}=\xi$ in (4.3) and then using (2.1), (2.3), we get

$$
\widehat{S}(\check{F}, \xi)=-\check{\lambda} \check{\eta}(\check{F}) .
$$

Thus from (3.6) and (4.4), it follows that

$$
\check{\lambda}=-(2 n+\check{\psi}) \text {. }
$$

Hence, (4.3) together with (4.5) leads to the following theorem.

Theorem 4.1. If an LP-Kenmotsu manifold $M$ with a connection $\widehat{\nabla}$ admits a Ricci soliton $(\check{g}, \xi, \check{\lambda})$, then $M$ is an $\eta$-Einstein manifold and its Ricci solition will be expanding, shrinking or steady according to $\check{\psi}<-2 n, \check{\psi}>-2 n$ or $\check{\psi}=-2 n$.

Now, assumig that $(\check{g}, U, \check{\lambda})$ is a Ricci soliton on an $L P$-Kenmotsu manifold with a connection $\widehat{\nabla}$ such that $U$ is pointwise collinear with $\xi$, i.e., $U=\beta \xi$, where $\beta$ is a function. Then (1.9) holds and we have

$$
\beta \check{g}\left(\widehat{\nabla}_{\check{E}} \xi, \check{F}\right)+(\check{E} \beta) \check{\eta}(\check{F})+\beta \check{g}\left(\check{E}, \widehat{\nabla}_{\check{F}} \xi\right)+(\check{F} \beta) \check{\eta}(\check{E})+2 \widehat{S}(\check{E}, \check{F})+2 \check{\lambda} \check{g}(\check{E}, \check{F})=0 \text {, }
$$

which in view of (3.7) and (1.6) becomes

(4.6) $-2 \beta[\check{g}(\check{E}, \check{F})+\check{\eta}(\check{E}) \check{\eta}(\check{F})]+(\check{E} \beta) \check{\eta}(\check{F})+(\check{F} \beta) \check{\eta}(\check{E})+2 \widehat{S}(\check{E}, \check{F})+2 \check{\lambda} \check{g}(\check{E}, \check{F})=0$.

Replacing $\check{F}$ by $\xi$ in (4.6) and using (2.1), (2.4) and (3.6), we find

$$
-(\check{E} \beta)+[(\xi \beta)+2(2 n+\check{\psi})+2 \check{\lambda}] \check{\eta}(\check{E})=0,
$$

which by taking $\check{E}=\xi$ and using (2.1) yields

$$
(\xi \beta)+(2 n+\check{\psi})+\check{\lambda}=0 .
$$

Combining the equations (4.7) and (4.8), we find

$$
d \beta=[(2 n+\check{\psi})+\check{\lambda}] \check{\eta} .
$$

Now, applying $d$ on (4.9), we get

$$
[(2 n+\check{\psi})+\check{\lambda}] \check{\eta}=0 \Longrightarrow \check{\lambda}=-(2 n+\check{\psi}), \quad d \check{\eta} \neq 0 .
$$


Thus, from (4.9) and (4.10), we obtain $d \beta=0$, i.e., $\beta$ is a constant. Therefore, (4.6) reduces to

$$
\widehat{S}(\check{E}, \check{F})=(\beta-\check{\lambda}) \check{g}(\check{E}, \check{F})+\beta \check{\eta}(\check{E}) \check{\eta}(\check{F}) .
$$

Hence, (4.10) together with (4.11) leads the following theorem.

Theorem 4.2. If an LP-Kenmotsu manifold $M$ with a connection $\widehat{\nabla}$ admits a Ricci soliton $(\check{g}, U, \breve{\lambda})$ such that $U$ is pointwise collinear with $\xi$, then $U$ is a constant multiple of $\xi$ and $M$ is an $\eta$-Einstein manifold and its Ricci solition will be expanding, shrinking or steady according to $\check{\psi}<-2 n, \check{\psi}>-2 n$ or $\check{\psi}=-2 n$.

\section{Ricci SOliton on $\check{\phi}$-SEMisymmetric $L P$-Kenmotsu MANifolds With a} CONNECTION $\widehat{\nabla}$

Definition 5.1. An $L P$-Kenmotsu manifold with a connection $\widehat{\nabla}$ is called $\check{\phi}$-projectively semisymmetric if (see [13]) $\widehat{P}(\check{E}, \check{F}) \cdot \check{\phi}=0$ for all $\check{E}, \check{F}$ on $M$.

Analogous to the equation (1.2), the projective curvature tensor with a connection $\bar{\nabla}$ is given by

$$
\widehat{P}(\check{E}, \check{F}) \check{W}=\widehat{R}(\check{E}, \check{F}) \check{W}-\frac{1}{2 n}[\widehat{S}(\check{F}, \check{W}) \check{E}-\widehat{S}(\check{E}, \check{W}) \check{F}] .
$$

Suppose that a $(2 n+1)$-dimensional $L P$-Kenmotsu manifold with a connection $\widehat{\nabla}$ is $\check{\phi}$-projectively semisymmetric, therefore

$$
(\widehat{P}(\check{E}, \check{F}) \cdot \check{\phi}) \check{W}=\widehat{P}(\check{E}, \check{F}) \check{\phi} \check{W}-\check{\phi} \widehat{P}(\check{E}, \check{F}) \check{W}=0,
$$

for all $\check{E}, \check{F}, \check{W}$ on $M$. From (5.1), we find

$$
\begin{aligned}
& \widehat{P}(\check{E}, \check{F}) \check{\phi} \check{W}=\widehat{R}(\check{E}, \check{F}) \check{\phi} \check{W}-\frac{1}{2 n}[\widehat{S}(\check{F}, \check{\phi} \check{W}) \check{E}-\widehat{S}(\check{E}, \check{\phi} \check{W}) \check{F}], \\
& \check{\phi} \widehat{P}(\check{E}, \check{F}) \check{W}=\check{\phi} \widehat{R}(\check{E}, \check{F}) \check{W}-\frac{1}{2 n}[\widehat{S}(\check{F}, \check{W}) \check{\phi} \check{E}-\widehat{S}(\check{E}, \check{W}) \check{\phi} \check{F}] .
\end{aligned}
$$

By combining (5.2), (5.3) and (5.4), we have

$$
\begin{aligned}
& \widehat{R}(\check{E}, \check{F}) \check{\phi} \check{W}-\check{\phi} \widehat{R}(\check{E}, \check{F}) \check{W}-\frac{1}{2 n}[\widehat{S}(\check{F}, \check{\phi} \check{W}) \check{E}-\widehat{S}(\check{E}, \check{\phi} \check{W}) \check{F}] \\
& +\frac{1}{2 n}[\widehat{S}(\check{F}, \check{W}) \check{\phi} \check{E}-\widehat{S}(\check{E}, \check{W}) \check{\phi} \check{F}]=0 .
\end{aligned}
$$

Taking $\check{F}=\xi$ in (5.5) and using (2.3), (3.5) and (3.6), we find

$$
-\check{g}(\check{E}, \check{\phi} \check{W}) \xi+\frac{1}{2 n} \widehat{S}(\check{E}, \check{\phi} \check{W}) \xi-\check{\eta}(\check{W}) \check{\phi} \check{E}-\check{\eta}(\check{W}) \check{\phi} 2 \check{E}+\frac{(2 n+\check{\psi})}{2 n} \check{\eta}(\check{W}) \check{\phi} \check{E}=0 .
$$

Taking inner product of the above equation with $\xi$ and making use of (2.1) and (2.3) yields $\widehat{S}(\check{E}, \check{\phi} \check{W})=2 n \check{g}(\check{E}, \check{\phi} \check{W})$, which by setting $\check{W}=\check{\phi} \check{W}$ and using $(2.2)$ gives

$$
\widehat{S}(\check{E}, \check{W})=2 n \check{g}(\check{E}, \check{W})-\check{\psi} \check{\eta}(\check{E}) \check{\eta}(\check{W}) \text {. }
$$


Now, taking $\check{W}=\xi$ in $(5.6)$, we find

$$
\widehat{S}(\check{E}, \xi)=(2 n+\check{\psi}) \check{\eta}(\check{E}) .
$$

Thus, from (4.4) and (5.7), we obtain

$$
\check{\lambda}=-(2 n+\check{\psi}) \text {. }
$$

Hence, (5.6) together with (5.8) leads to the following theorem.

Theorem 5.1. If a $(2 n+1)$-dimensional LP-Kenmotsu manifold $M$ with a connection $\widehat{\nabla}$ admitting Ricci soliton is $\check{\phi}$-projectively semisymmetric, then $M$ is an $\eta$-Einstein manifold and its Ricci solition will be expanding, shrinking or steady according to $\breve{\psi}<-2 n, \breve{\psi}>-2 n$ or $\breve{\psi}=-2 n$.

Definition 5.2. An $L P$-Kenmotsu manifold with a connection $\widehat{\nabla}$ is called $\check{\phi}$-concircularly semisymmetric if $\widehat{V}(\check{E}, \check{F}) \cdot \check{\phi}=0$ for all $\check{E}, \check{F}$ on $M$.

Analogous to the equation (1.3), the concircular curvature tensor with a connection $\widehat{\nabla}$ is given by

$$
\widehat{V}(\check{E}, \check{F}) \check{W}=\widehat{R}(\check{E}, \check{F}) \check{W}-\frac{\widehat{r}}{2 n(2 n+1)}[\check{g}(\check{F}, \check{W}) \check{E}-\check{g}(\check{E}, \check{W}) \check{F}] .
$$

Suppose that a $(2 n+1)$-dimensional $L P$-Kenmotsu manifold with a connection $\widehat{\nabla}$ is $\check{\phi}$-concircularly semisymmetric, therefore

$$
(\widehat{V}(\check{E}, \check{F}) \cdot \check{\phi}) \check{W}=\widehat{V}(\check{E}, \check{F}) \check{\phi} \check{W}-\check{\phi} \widehat{V}(\check{E}, \check{F}) \check{W}=0,
$$

for all $\check{E}, \check{F}, \check{W}$ on $M$. From (5.9), it follows that

$$
\begin{aligned}
& \widehat{V}(\check{E}, \check{F}) \check{\phi} \check{W}=\widehat{R}(\check{E}, \check{F}) \check{\phi} \check{W}-\frac{\widehat{r}}{2 n(2 n+1)}[\check{g}(\check{F}, \check{\phi} \check{W}) \check{E}-\check{g}(\check{E}, \check{\phi} \check{W}) \check{F}], \\
& \check{\phi} \widehat{V}(\check{E}, \check{F}) \check{W}=\check{\phi} \widehat{R}(\check{E}, \check{F}) \check{W}-\frac{\widehat{r}}{2 n(2 n+1)}[\check{g}(\check{F}, \check{W}) \check{\phi} \check{E}-\check{g}(\check{E}, \check{W}) \check{\phi} \check{F}] .
\end{aligned}
$$

Combining (5.10), (5.11) and (5.12), we have

$$
\begin{aligned}
& \widehat{R}(\check{E}, \check{F}) \check{\phi} \check{W}-\check{\phi} \widehat{R}(\check{E}, \check{F}) \check{W}-\frac{\widehat{r}}{2 n(2 n+1)}[\check{g}(\check{F}, \check{\phi} \check{W}) \check{E}-\check{g}(\check{E}, \check{\phi} \check{W}) \check{F}] \\
& +\frac{\widehat{r}}{2 n(2 n+1)}[\check{g}(\check{F}, \check{W}) \check{\phi} \check{E}-\check{g}(\check{E}, \check{W}) \check{\phi} \check{F}]=0,
\end{aligned}
$$

which, by taking $\check{F}=\xi$ and using (2.3), (2.4) and (3.5), takes the form

$$
\left[\frac{\widehat{r}}{2 n(2 n+1)}-1\right] \check{g}(\check{E}, \check{\phi} \check{W}) \xi+\left[\frac{\widehat{r}}{2 n(2 n+1)}-1\right] \check{\eta}(\check{W}) \check{\phi} \check{E}+\check{\eta}(\check{W}) \check{\phi} \check{\phi}^{2} \check{E}=0 .
$$

Taking inner product of (5.13) with $\xi$ and making use of (2.1) and (2.3), we get

$$
\widehat{r}=2 n(2 n+1), \quad \check{g}(\check{E}, \check{\phi} \check{W}) \neq 0 .
$$

This leads to the following theorem. 
Theorem 5.2. If a $(2 n+1)$-dimensional LP-Kenmotsu manifold with a connection $\widehat{\nabla}$ is $\check{\phi}$-concirculary semisymmetric, then the scalar curvature is constant.

Definition 5.3. An LP-Kenmotsu manifold with a connection $\widehat{\nabla}$ is called $\check{\phi}$-conformally semisymmetric if $\widehat{C}(\check{E}, \check{F}) \cdot \check{\phi}=0$ for all $\check{E}, \check{F}$ on $M$.

Analogous to the equation (1.4), the conformal curvature tensor with a connection $\widehat{\nabla}$ is given by

$$
\begin{aligned}
\widehat{C}(\check{E}, \check{F}) \check{W}= & \widehat{R}(\check{E}, \check{F}) \check{W}-\frac{1}{(2 n-1)}[\widehat{S}(\check{F}, \check{W}) \check{E}-\widehat{S}(\check{E}, \check{W}) \check{F} \\
& +\check{g}(\check{F}, \check{W}) \widehat{Q} \check{E}-\check{g}(\check{E}, \check{W}) \widehat{Q} \check{F}]+\frac{\widehat{r}}{2 n(2 n-1)}[\check{g}(\check{F}, \check{W}) \check{E}-\check{g}(\check{E}, \check{W}) \check{F}] .
\end{aligned}
$$

Suppose that a $(2 n+1)$-dimensional $L P$-Kenmotsu manifold with a connection $\widehat{\nabla}$ is $\check{\phi}$-conformally semisymmetric, therefore

$$
(\widehat{C}(\check{E}, \check{F}) \cdot \check{\phi}) \check{W}=\widehat{C}(\check{E}, \check{F}) \check{\phi} \check{W}-\check{\phi} \widehat{C}(\check{E}, \check{F}) \check{W}=0,
$$

for all $\check{E}, \check{F}, \check{W}$ on $M$. From (5.14), it follows that

$$
\begin{aligned}
\widehat{C}(\check{E}, \check{F}) \check{\phi} \check{W}= & \widehat{R}(\check{E}, \check{F}) \check{\phi} \check{W}-\frac{1}{(2 n-1)}[\widehat{S}(\check{F}, \check{\phi} \check{W}) E \\
& -\widehat{S}(\check{E}, \check{\phi} \check{W}) \check{F}+\check{g}(\check{F}, \check{\phi} \check{W}) \widehat{Q} \check{E}-\check{g}(\check{E}, \check{\phi} \check{W}) \widehat{Q} \check{F}] \\
& +\frac{\widehat{r}}{2 n(2 n-1)}[\check{g}(\check{F}, \check{\phi} \check{W}) \check{E}-\check{g}(\check{E}, \check{\phi} \check{W}) \check{F}], \\
\check{\phi} \widehat{C}(\check{E}, \check{F}) \check{W}= & \check{\phi} \widehat{R}(\check{E}, \check{F}) \check{W}-\frac{1}{(2 n-1)}[\widehat{S}(\check{F}, \check{W}) \check{\phi} \check{E} \\
& -\widehat{S}(\check{E}, \check{W}) \check{\phi} \check{F}+\check{g}(\check{F}, \check{W}) \check{\phi} \widehat{Q} \check{E}-\check{g}(\check{E}, \check{W}) \check{\phi} \widehat{Q} \check{F}] \\
& +\frac{\widehat{r}}{2 n(2 n-1)}[\check{g}(\check{F}, \check{W}) \check{\phi} \check{E}-\check{g}(\check{E}, \check{W}) \check{\phi} \check{F}] .
\end{aligned}
$$

Combining (5.15), (5.16) and (5.17), we have

$$
\begin{aligned}
& \widehat{R}(\check{E}, \check{F}) \check{\phi} \check{W}-\check{\phi} \widehat{R}(\check{E}, \check{F}) \check{W}-\frac{1}{(2 n-1)}[\widehat{S}(\check{F}, \check{\phi} \check{W}) \check{E}-\widehat{S}(\check{E}, \check{\phi} \check{W}) \check{F} \\
& +\check{g}(\check{F}, \check{\phi} \check{W}) \widehat{Q} \check{E}-\check{g}(\check{E}, \check{\phi} \check{W}) \widehat{Q} \check{F}]+\frac{1}{(2 n-1)}[\widehat{S}(\check{F}, \check{W}) \check{\phi} \check{E}-\widehat{S}(\check{E}, \check{W}) \check{\phi} \check{F} \\
& +\check{g}(\check{F}, \check{W}) \check{\phi} \widehat{Q} \check{E}-\check{g}(\check{E}, \check{W}) \check{\phi} \widehat{Q} \check{F}]+\frac{\widehat{r}}{2 n(2 n-1)}[\check{g}(\check{F}, \check{\phi} \check{W}) \check{E}-\check{g}(\check{E}, \check{\phi} \check{W}) \check{F}] \\
& -\frac{\widehat{r}}{2 n(2 n-1)}[\check{g}(\check{F}, \check{W}) \check{\phi} \check{E}-\check{g}(\check{E}, \check{W}) \check{\phi} \check{F}]=0,
\end{aligned}
$$


which by replacing $\check{E}=\xi$ and making use of (2.3), (2.4), (3.5), (3.6) and (3.8) takes the form

$$
\begin{aligned}
& {\left[\frac{2 n+\check{\psi}}{2 n-1}-\frac{\widehat{r}}{2 n(2 n-1)}-1\right](\check{g}(\check{E}, \check{\phi} \check{W}) \xi+\check{\eta}(\check{W}) \check{\phi} E)+\frac{1}{(2 n-1)} \widehat{S}(\check{E}, \check{\phi} \check{W}) \xi} \\
& +\frac{1}{(2 n-1)} \check{\eta}(\check{W}) \check{\phi} \widehat{Q} \check{E}-\check{\eta}(\check{W}) \check{\phi} 2 \check{E}=0 .
\end{aligned}
$$

Now, taking inner product of (5.18) with $\xi$ and making use of (2.1) and (2.3), we obtain

$$
\widehat{S}(\check{E}, \check{\phi} \check{W})=\left[(2 n-1)+\frac{\widehat{r}}{2 n}-(2 n+\check{\psi})\right] \check{g}(\check{E}, \check{\phi} \check{W}) .
$$

By replacing $\check{W}$ by $\check{\phi} \check{W}$ in (5.19) and then using (2.2), (2.4), (3.6), we get

$$
\begin{aligned}
\widehat{S}(\check{E}, \check{W})= & {\left[(2 n-1)+\frac{\widehat{r}}{2 n}-(2 n+\check{\psi})\right] \check{g}(\check{E}, \check{W}) } \\
& +\left[(2 n-1)+\frac{\widehat{r}}{2 n}-2(2 n+\check{\psi})\right] \check{\eta}(\check{E}) \check{\eta}(\check{W}) .
\end{aligned}
$$

Taking $\check{W}=\xi$ in $(5.20)$, we find

$$
\widehat{S}(\check{E}, \xi)=(2 n+\check{\psi}) \check{\eta}(\check{E})
$$

Thus, from (4.4) and (5.21), we obtain

$$
\check{\lambda}=-(2 n+\check{\psi}) \text {. }
$$

Hence, (5.20) together with (5.22) leads to the following theorem.

Theorem 5.3. If a $(2 n+1)$-dimensional LP-Kenmotsu manifold $M$ with a connection $\widehat{\nabla}$ admitting Ricci soliton is $\check{\phi}$-conformally semisymmetric, then $M$ is an $\eta$-Einstein manifold and its Ricci solition will be expanding, shrinking or steady according to $\check{\psi}<-2 n, \check{\psi}>-2 n$ or $\breve{\psi}=-2 n$.

Definition 5.4. An $L P$-Kenmotsu manifold with a connection $\widehat{\nabla}$ is called $\check{\phi}$ - $D$ conformally semisymmetric if $\widehat{B}(\check{E}, \check{F}) \cdot \check{\phi}=0$ for all $\check{E}, \check{F}$ on $M$.

Analogous to the equation (1.5), the $D$-conformal curvature tensor with a connection $\widehat{\nabla}$ is given by

$$
\begin{aligned}
\widehat{B}(\check{E}, \check{F}) \check{W}= & \widehat{R}(\check{E}, \check{F}) \check{W}+\frac{1}{2(n-1)}[\widehat{S}(\check{E}, \check{W}) \check{F}-\widehat{S}(\check{F}, \check{W}) \check{E} \\
& +\check{g}(\check{E}, \check{W}) \widehat{Q} \check{F}-\check{g}(\check{F}, \check{W}) \widehat{Q} \check{E}-\widehat{S}(\check{E}, \check{W}) \check{\eta}(\check{F}) \xi \\
& +\widehat{S}(\check{F}, \check{W}) \check{\eta}(\check{E}) \xi-\check{\eta}(\check{E}) \check{\eta}(\check{W}) \widehat{Q} \check{F}+\check{\eta}(\check{F}) \check{\eta}(\check{W}) \widehat{Q} \check{E}] \\
& -\frac{\widehat{k}-2}{2(n-1)}[\check{g}(\check{E}, \check{W}) \check{F}-\check{g}(\check{F}, \check{W}) \check{E}]+\frac{\widehat{k}}{2(n-1)}[\check{g}(\check{E}, \check{W}) \check{\eta}(\check{F}) \xi \\
& -\check{g}(\check{F}, \check{W}) \check{\eta}(\check{E}) \xi+\check{\eta}(\check{E}) \check{\eta}(\check{W}) \check{F}-\check{\eta}(\check{F}) \check{\eta}(\check{W}) \check{E}],
\end{aligned}
$$


where $\widehat{k}=\frac{\widehat{r}+4 n}{(2 n-1)}$.

Suppose that a $(2 n+1)$-dimensional $L P$-Kenmotsu manifold with a connection $\widehat{\nabla}$ is $\check{\phi}$-D-conformally semisymmetric, therefore

$$
(\widehat{B}(\check{E}, \check{F}) \cdot \check{\phi}) \check{W}=\widehat{B}(\check{E}, \check{F}) \check{\phi} \check{W}-\check{\phi} \widehat{B}(\check{E}, \check{F}) \check{W}=0
$$

for all $\check{E}, \check{F}, \check{W}$ on $M$. From (5.23), it follows that

$$
\begin{aligned}
\widehat{B}(\check{E}, \check{F}) \check{\phi} \check{W}= & \widehat{R}(\check{E}, \check{F}) \check{\phi} \check{W}+\frac{1}{2(n-1)}[\widehat{S}(\check{E}, \check{\phi} \check{W}) \check{F}-\widehat{S}(\check{F}, \check{\phi} \check{W}) \check{E}+\check{g}(\check{E}, \check{\phi} \check{W}) \widehat{Q} \check{F} \\
& -\check{g}(\check{F}, \check{\phi} \check{W}) \widehat{Q} \check{E}-\widehat{S}(\check{E}, \check{\phi} \check{W}) \check{\eta}(\check{F}) \xi+\widehat{S}(\check{F}, \check{\phi} \check{W}) \check{\eta}(\check{E}) \xi] \\
& -\frac{\widehat{k}-2}{2(n-1)}[\check{g}(\check{E}, \check{\phi} \check{W}) \check{F}-\check{g}(\check{F}, \check{\phi} \check{W}) \check{E}]+\frac{\check{k}}{2(n-1)}[\check{g}(\check{E}, \check{\phi} \check{W}) \check{\eta}(\check{F}) \xi \\
& -\check{g}(\check{F}, \check{\phi} \check{W}) \check{\eta}(\check{E}) \xi],
\end{aligned}
$$

$$
\begin{aligned}
\check{\phi} \widehat{B}(\check{E}, \check{F}) W= & \check{\phi} \widehat{R}(\check{E}, \check{F}) \check{W}+\frac{1}{2(n-1)}[\widehat{S}(\check{E}, \check{W}) \check{\phi} \check{F}-\widehat{S}(\check{F}, \check{W}) \check{\phi} \check{E} \\
& +\check{g}(\check{E}, \check{W}) \check{\phi} \widehat{Q} \check{F}-\check{g}(\check{F}, \check{W}) \check{\phi} \widehat{Q} \check{E}-\check{\eta}(\check{E}) \check{\eta}(\check{W}) \check{\phi} \widehat{\phi} \check{F} \\
& +\check{\eta}(\check{F}) \check{\eta}(\check{W}) \check{\phi} \widehat{\phi} \check{E}]-\frac{\widehat{k}-2}{2(n-1)}[\check{g}(\check{E}, \check{W}) \check{\phi} \check{F}-\check{g}(\check{F}, \check{W}) \check{\phi} \check{E}] \\
& +\frac{\widehat{k}}{2(n-1)}[\check{\eta}(\check{E}) \check{\eta}(\check{W}) \check{\phi} \check{F}-\check{\eta}(\check{F}) \check{\eta}(\check{W}) \check{\phi} \check{E}] .
\end{aligned}
$$

Combining (5.24), (5.25) and (5.26), we have

$$
\begin{aligned}
& \widehat{R}(\check{E}, \check{F}) \check{\phi} \check{W}-\check{\phi} \widehat{R}(\check{E}, \check{F}) \check{W}+\frac{1}{2(n-1)}[\widehat{S}(\check{E}, \check{\phi} \check{W}) \check{F}-\widehat{S}(\check{F}, \check{\phi} \check{W}) \check{E} \\
& +\check{g}(\check{E}, \check{\phi} \check{W}) \widehat{Q} \check{F}-\check{g}(\check{F}, \check{\phi} \check{W}) \widehat{Q} \check{E}-\widehat{S}(\check{E}, \check{\phi} \check{W}) \check{\eta}(\check{F}) \xi+\widehat{S}(\check{F}, \check{\phi} \check{W}) \check{\eta}(\check{E}) \xi] \\
& -\frac{1}{2(n-1)}[\widehat{S}(\check{E}, \check{W}) \check{\phi} \check{F}-\widehat{S}(\check{F}, \check{W}) \check{\phi} \check{E}+\check{g}(\check{E}, \check{W}) \check{\phi} \widehat{Q} \check{F}-\check{g}(\check{F}, \check{W}) \check{\phi} \widehat{Q} \check{E} \\
& -\check{\eta}(\check{E}) \check{\eta}(\check{W}) \check{\phi} \widehat{Q} \check{F}+\check{\eta}(\check{F}) \check{\eta}(\check{W}) \check{\phi} \widehat{Q} \check{E}]-\frac{\widehat{k}-2}{2(n-1)}[\check{g}(\check{E}, \check{\phi} \check{W}) \check{F}-\check{g}(\check{F}, \check{\phi} \check{W}) \check{E}] \\
& +\frac{\widehat{k}-2}{2(n-1)}[\check{g}(\check{E}, \check{W}) \check{\phi} \check{F}-\check{g}(\check{F}, \check{W}) \check{\phi} \check{E}]+\frac{\widehat{k}}{2(n-1)}[\check{g}(\check{E}, \check{\phi} \check{W}) \check{\eta}(\check{F}) \\
& -\check{g}(\check{F}, \check{\phi} \check{W}) \check{\eta}(\check{E})] \xi-\frac{\widehat{k}}{2(n-1)}[\check{\eta}(\check{E}) \check{\eta}(\check{W}) \check{\phi} \check{F}-\check{\eta}(\check{F}) \check{\eta}(\check{W}) \check{\phi} \check{E}]=0 .
\end{aligned}
$$


By taking $\check{F}=\xi$ in (5.27) and then using (2.1), (2.3), (3.5), (3.6) and (3.8) takes the form

$$
\begin{aligned}
& \frac{4+\check{\psi}-2 \widehat{k}}{2(n-1)}[\check{g}(\check{E}, \check{\phi} \check{W}) \xi+\check{\eta}(\check{W}) \check{\phi} \check{E}]+\frac{1}{n-1} \widehat{S}(\check{E}, \check{\phi} \check{W}) \xi-\check{\eta}(\check{W}) \check{\phi}^{2} \check{E} \\
& +\frac{1}{n-1} \check{\eta}(\check{W}) \check{\phi} \widehat{Q} \check{E}=0 .
\end{aligned}
$$

Inner product of (5.28) with $\xi$ and making use of (2.1) and (2.3) gives

$$
\widehat{S}(\check{E}, \check{\phi} \check{W})=\left[\frac{\breve{\psi}}{2}+2-\widehat{k}\right] \check{g}(\check{E}, \check{\phi} \check{W}) .
$$

Now, we replace $\check{W}$ by $\check{\phi} \check{W}$ in (5.29) and using (2.2), (2.4) and (3.6), we get

$$
\widehat{S}(\check{E}, \check{W})=\left[\frac{\check{\psi}}{2}+2-\widehat{k}\right] \check{g}(\check{E}, \check{W})-\left[\frac{\check{\psi}}{2}+\widehat{k}+2 n-2\right] \check{\eta}(\check{E}) \check{\eta}(\check{W}) .
$$

Taking $\check{W}=\xi$ in $(5.30)$, we find

$$
\widehat{S}(\check{E}, \xi)=(2 n+\check{\psi}) \check{\eta}(\check{E})
$$

Thus, from (4.4) and (5.31), we obtain

$$
\check{\lambda}=-(2 n+\check{\psi}) \text {. }
$$

Hence, (5.30) together with (5.32) leads to the following theorem.

Theorem 5.4. If a $(2 n+1)$-dimensional LP-Kenmotsu manifold $M$ with a connection $\widehat{\nabla}$ admitting Ricci soliton is $\check{\phi}$-D-conformally semisymmetric, then $M$ is an $\eta$-Einstein manifold and its Ricci solition will be expanding, shrinking or steady according to $\check{\psi}<-2 n, \check{\psi}>-2 n$ or $\check{\psi}=-2 n$.

Example 5.1. Let on a 3-dimensional manifold $M=\left\{\left(\check{w}_{1}, \check{w}_{2}, \check{w}_{3}\right) \in R^{3}: w>0\right\}$, where $\left(\check{w}_{1}, \check{w}_{2}, \check{w}_{3}\right)$ are the standard coordinates of $R^{3}$, the linearly independent vector fields that at each point of $M$ are given by

$$
v^{1}=\frac{\check{w}_{3} \partial}{\partial \check{w}_{1}}, \quad v^{2}=\frac{w \partial}{\partial \check{w}_{2}}, \quad v^{3}=\frac{w \partial}{\partial \check{w}_{3}}=\xi
$$

Suppose the Lorentzian metric $\breve{g}$ is defined by

$$
\check{g}\left(v^{1}, v^{1}\right)=\check{g}\left(v^{2}, v^{2}\right)=1, \quad \check{g}\left(v^{3}, v^{3}\right)=-1, \quad \check{g}\left(v^{1}, v^{2}\right)=\check{g}\left(v^{2}, v^{3}\right)=\check{g}\left(v^{1}, v^{3}\right)=0 .
$$

Suppose the 1-form $\check{\eta}$ is defined by $\check{\eta}(\check{E})=\check{g}\left(\check{E}, v^{3}\right)=\check{g}(\check{E}, \xi)$ for all $\check{E}$ on $M$, and the $(1,1)$-tensor field $\check{\phi}$ is defined by

$$
\check{\phi} v^{1}=-v^{1}, \quad \check{\phi} v^{2}=-v^{2}, \quad \check{\phi} v^{3}=0 .
$$

Then, using the linearity of $\check{g}$ and $\check{\phi}$, we have

$$
\check{\eta}(\xi)=-1, \quad \check{\phi}^{2} \check{E}=\check{E}+\check{\eta}(\check{E}) \xi, \quad \check{g}(\check{\phi} \check{E}, \check{\phi} \check{F})=\check{g}(\check{E}, \check{F})+\check{\eta}(\check{E}) \check{\eta}(\check{F}),
$$


for all $\check{E}, \check{F}$ on $M$. Thus, $(\check{\phi}, \xi, \check{\eta}, \check{g})$ defines a Lorentzian almost paracontact metric structure on $M$. Also, we have

$$
\left[v^{1}, v^{2}\right]=0, \quad\left[v^{1}, v^{3}\right]=-v^{1}, \quad\left[v^{2}, v^{3}\right]=-v^{2} .
$$

From the Koszul's formula for $\check{g}$, we calculate

$$
\begin{aligned}
& \check{\nabla}_{v^{1}} v^{1}=-v^{3}, \quad \check{\nabla}_{v^{1}} v^{2}=0, \quad \check{\nabla}_{v^{1}} v^{3}=-v^{1}, \quad \check{\nabla}_{v^{2}} v^{1}=0, \\
& \check{\nabla}_{v^{2}} v^{2}=-v^{3}, \quad \check{\nabla}_{v^{2}} v^{3}=-v^{2}, \quad \check{\nabla}_{v^{3}} v^{1}=0, \quad \check{\nabla}_{v^{3}} v^{2}=0, \quad \check{\nabla}_{v^{3}} v^{3}=0 .
\end{aligned}
$$

Also, one can easily verify that

$$
\check{\nabla}_{\check{E}} \xi=-\check{E}-\check{\eta}(\check{E}) \xi \text { and }\left(\check{\nabla}_{\check{E}} \check{\phi}\right) \check{F}=-\check{g}(\check{\phi} \check{E}, \check{F}) \xi-\check{\eta}(\check{F}) \check{\phi} \check{E} \text {. }
$$

Therefore, $M$ is an LP-Kenmotsu manifold. From (1.1), (5.33) and (5.34), we obtain

$$
\begin{aligned}
& \check{R}\left(v^{1}, v^{2}\right) v^{1}=-v^{2}, \quad \check{R}\left(v^{2}, v^{3}\right) v^{1}=0, \quad \check{R}\left(v^{1}, v^{3}\right) v^{1}=-v^{3}, \\
& \check{R}\left(v^{1}, v^{2}\right) v^{2}=v^{1}, \quad \check{R}\left(v^{1}, v^{3}\right) v^{2}=0, \quad \check{R}\left(v^{2}, v^{3}\right) v^{2}=-v^{3}, \\
& \check{R}\left(v^{1}, v^{2}\right) v^{3}=0, \quad \check{R}\left(v^{1}, v^{3}\right) v^{3}=-v^{1}, \quad \check{R}\left(v^{2}, v^{3}\right) v^{3}=-v^{2},
\end{aligned}
$$

from which it is clear that $\check{R}(\check{E}, \check{F}) \check{W}=\check{g}(\check{F}, \check{W}) \check{E}-\check{g}(\check{E}, \check{W}) \check{F}$. Hence, $(M, \check{\phi}, \xi, \check{\eta}, \check{g})$ is an $L P$-Kenmotsu manifold of unit constant curvature. By virtue of (1.8) and (5.35), we obtain

$$
\begin{aligned}
& \widehat{\nabla}_{v^{1}} v^{1}=-v^{3}, \quad \widehat{\nabla}_{v^{2}} v^{1}=0, \quad \widehat{\nabla}_{v^{3}} v^{1}=0, \quad \widehat{\nabla}_{v^{1}} v^{2}=0, \quad \widehat{\nabla}_{v^{2}} v^{2}=-v^{3}, \\
& \widehat{\nabla}_{v^{3}} v^{2}=0, \quad \widehat{\nabla}_{v^{1}} v^{3}=0, \quad \widehat{\nabla}_{v^{2}} v^{3}=0, \quad \widehat{\nabla}_{v^{3}} v^{3}=0 .
\end{aligned}
$$

From (3.2) and (5.35), we can easily obtain

$$
\begin{array}{ll}
\widehat{R}\left(v^{1}, v^{2}\right) v^{1}=0, & \widehat{R}\left(v^{1}, v^{3}\right) v^{1}=-v^{3}, \quad \widehat{R}\left(v^{2}, v^{3}\right) v^{1}=0, \\
\widehat{R}\left(v^{1}, v^{2}\right) v^{2}=0, & \widehat{R}\left(v^{1}, v^{3}\right) v^{2}=0, \quad \widehat{R}\left(v^{2}, v^{3}\right) v^{2}=-v^{3}, \\
\widehat{R}\left(v^{1}, v^{2}\right) v^{3}=0, & \widehat{R}\left(v^{1}, v^{3}\right) v^{3}=0, \quad \widehat{R}\left(v^{2}, v^{3}\right) v^{3}=0 .
\end{array}
$$

From (5.35) and (5.36), we calculate the Ricci tensors as follows:

$$
\check{S}\left(v^{1}, v^{1}\right)=\check{S}\left(v^{2}, v^{2}\right)=2, \quad \check{S}\left(v^{3}, v^{3}\right)=-2,
$$

and

$$
\widehat{S}\left(v^{1}, v^{1}\right)=\widehat{S}\left(v^{2}, v^{2}\right)=1, \quad \widehat{S}\left(v^{3}, v^{3}\right)=0 .
$$

Therefore, we find $\check{r}=6$ and $\widehat{r}=2$, where $\breve{\psi}=-2$. Hence, (3.4) is satisfied. From (2.5), (1.6) and (1.7), we find

$$
\begin{aligned}
\check{\Phi}\left(v^{1}, v^{1}\right) & =\check{\Phi}\left(v^{2}, v^{2}\right)=-1, \quad \check{\Phi}\left(v^{3}, v^{3}\right)=0, \\
\check{T}\left(v^{i}, v^{j}\right) & =0, \quad i=j=1,2,3, \\
\check{T}\left(v^{1}, v^{2}\right) & =0, \quad \check{T}\left(v^{1}, v^{3}\right)=v^{1}, \quad \check{T}\left(v^{2}, v^{3}\right)=v^{2}, \\
\left(\widehat{\nabla}_{v^{1}} \check{g}\right)\left(v^{1}, v^{3}\right) & =\left(\widehat{\nabla}_{v^{2}} \check{g}\right)\left(v^{2}, v^{3}\right)=-1, \quad\left(\widehat{\nabla}_{v^{3}} \check{g}\right)\left(v^{1}, v^{2}\right)=0,
\end{aligned}
$$


respectively. Thus, the connection $\widehat{\nabla}$ defined on $M$ is a QSNM. Now, by putting $F=W=v^{i}$ in (4.3) and summing up, we find $2=3(1-\check{\lambda})-1$ implies $\check{\lambda}=0$. Thus, a Ricci soliton on an $L P$-Kenmotsu manifold with a connection $\widehat{\nabla}$ is steady for $\check{\psi}=-2 n=-2$.

\section{REFERENCES}

[1] A. Haseeb and R. Prasad, Certain results on Lorentzian para-Kenmotsu manifolds, Bol. Soc. Parana. Mat. DOI 10.5269/bspm.40607.

[2] A. Prakash and D. Narain, On a quarter-symmetric non-metric connection in a Lorentzian para-Sasakian manifold, Int. Electron. J. Geom. 4 (2011), 129-137.

[3] A. K. Mondal and U. C. De, Quarter-symmetric non-metric connection on P-Sasakian manifolds, ISRN Geometry (2012), Article ID 659430, 14 pages.

[4] D. E. Blair, Contact Manifolds in Riemannian Geometry, Lecture Notes in Mathematics 509, Springer-Verlag, Berlin, New York, 1976.

[5] G. Chuman, On D-conformal curvature tensor, Tensor (N. S.) 46 (1983), 125-129.

[6] K. Yano and M. Kon, Structures on Manifolds, Series in Pure Math. 3, World Science, Singapore, 1984.

[7] M. Ahmad, C. Özgür and A. Haseeb, Hypersurfaces of an almost r-paracontact Riemannian manifold endowed with a quarter symmetric non-metric connection, Kyungpook Math. J. 49 (2009), 533-543.

[8] P. Alegre, Slant submanifolds of Lorentzian Sasakian and Para Sasakian manifolds, Taiwanese J. Math. 17 (2013), 897-910.

[9] R. S. Hamilton, The Ricci flow on surfaces, in: Mathematics and General Relativity, Contemp. Math. 71, American Math. Soc., Providence, 1988, 237-262.

[10] R. S. Hamilton, Three-manifolds with positive Ricci curvature, J. Differential Geom. 17 (1982), $255-306$.

[11] S. Golab, On semi-symmetric and quarter-symmetric linear connections, Tensor (N. S.) 29 (1975), 249-254.

[12] U. C. De and A. K. Mondal, Hypersurfaces of Kenmotsu manifolds endowed with a quarter symmetric non-metric connection, Kuwait J. Sci. 39 (2012), 43-56.

[13] U. C. De and P. Majhi, ф-semisymmetric generalized Sasakian space-forms, Arab J. Math. Sci. 21 (2015), 170-178.

${ }^{1}$ Department of Mathematics and Astronomy,

UNIVERSITY OF LUCKNOW,

LUCKNOW-226007, INDIA.

Email address: rp.manpur@rediffmail.com,

Email address: ugautamarya@gmail. $\mathrm{com}^{3}$

${ }^{2}$ Department of Mathematics,

FaCUlty of Science, Jazan University,

JaZAn-2097, Kingdom of Saudi Arabia.

Email address: malikhaseeb80@gmail.com, haseeb@jazanu.edu.sa 